\title{
Synergistic Effects of Nonthermal Plasma and Disinfecting Agents against Dental Biofilms In Vitro
}

\author{
Ina Koban, ${ }^{1}$ Marie Henrike Geisel, ${ }^{1,2}$ Birte Holtfreter, ${ }^{1}$ Lukasz Jablonowski, ${ }^{1}$ \\ Nils-Olaf Hübner, ${ }^{3,4}$ Rutger Matthes, ${ }^{3}$ Kai Masur, ${ }^{5}$ Klaus-Dieter Weltmann, ${ }^{5}$ \\ Axel Kramer, ${ }^{3}$ and Thomas Kocher ${ }^{1}$ \\ ${ }^{1}$ Unit of Periodontology, Department of Restorative Dentistry, Periodontology and Endodontology, \\ Ernst-Moritz-Arndt University Greifswald, Walther-Rathenau-Straße 49a, 17487 Greifswald, Germany \\ ${ }^{2}$ Institute for Medical Informatics, Biometry and Epidemiology, University of Duisburg-Essen, Hufelandstrasse 55, \\ 45122 Essen, Germany \\ ${ }^{3}$ Institute for Hygiene and Environmental Medicine, Ernst-Moritz-Arndt University Greifswald, Walther-Rathenau-Straße 49a, \\ 17475 Greifswald, Germany \\ ${ }^{4}$ Robert Koch Institut, Nordufer 20, 13353 Berlin, Germany \\ ${ }^{5}$ Leibniz Institute for Plasma Science and Technology, Felix-Hausdorff-Straße 2, 17489 Greifswald, Germany
}

Correspondence should be addressed to Ina Koban; ina.koban@uni-greifswald.de

Received 14 July 2013; Accepted 7 August 2013

Academic Editors: H. S. Cardash and G. H. Sperber

Copyright (C) 2013 Ina Koban et al. This is an open access article distributed under the Creative Commons Attribution License, which permits unrestricted use, distribution, and reproduction in any medium, provided the original work is properly cited.

\begin{abstract}
Aim. Dental biofilms play a major role in the pathogenesis of many dental diseases. In this study, we evaluated the synergistic effect of atmospheric pressure plasma and different agents in dentistry on the reduction of biofilms. Methods and Results. We used monospecies (S. mutans) and multispecies dental biofilm models grown on titanium discs in vitro. After treatment with one of the agents, the biofilms were treated with plasma. Efficacy of treatment was determined by the number of colony forming units (CFU) and by live-dead staining. For S. mutans biofilms no colonies could be detected after treatment with $\mathrm{NaOCl}_{\text {or }} \mathrm{H}_{2} \mathrm{O}_{2}$. For multispecies biofilms the combination with plasma achieved a higher CFU reduction than each agent alone. We found an additive antimicrobial effect between argon plasma and agents irrespective of the treatment order with cultivation technique. For EDTA and octenidine, antimicrobial efficacy assessed by live-dead staining differed significantly between the two treatment orders $(P<0.05)$. Conclusions. The effective treatment of dental biofilms on titanium discs with atmospheric pressure plasma could be increased by adding agents in vitro.
\end{abstract}

\section{Introduction}

Plasma is the fourth state of matter besides the solid, liquid, and gaseous states. It is an ionized gas consisting of ions, a broad spectrum of radicals, ultraviolet irradiation, electric fields, and ozone, which are responsible for its antimicrobial efficacy [1].

Atmospheric pressure plasma is also called nonthermal plasma, because it can achieve body temperature [2]. This allows medical application to humans by small plasma hand device on humans [3]. Plasma medicine is a new scientific field, and many research groups investigated various applications, for example, to the treatment of dental diseases like periodontitis [4], peri-implantitis [5], and caries and denture stomatitis [6] as well as dermatological diseases and chronic wounds $[1,7,8]$. Most applications were based on the antimicrobial effect of plasma to disinfect the skin, implants, and other medical devices. To date antiseptics have been commonly applied in these cases.

Plasma is especially interesting for fields with a dissatisfactory standard therapy or where an effective therapy does not exist, for example, peri-implantitis therapy in dentistry. 
The antimicrobial efficacy of plasma could be increased by raising the electrical input power $[9,10]$. However, plasma should also be tissue tolerable and applicable to humans. As with any active substance the balance between efficacy and tolerability needs to be found.

To enhance plasma effects without an increased input power, we investigated possible synergistic effects between atmospheric pressure plasma and different antiseptics including chlorhexidine, octenidine, and polyhexanide as well as the chemicals sodium hypochlorite and hydrogen peroxide, often used in dental practice, and the chelating agent EDTA.

\section{Material and Methods}

We used three different dental biofilm models grown on titanium, the typical dental implant material. Titanium discs were machined and had a diameter of $5 \mathrm{~mm}$ and $1 \mathrm{~mm}$ thickness (Straumann, Basel, Switzerland).

2.1. Monospecies Biofilm Streptococcus mutans. Streptococcus mutans (S. mutans DSM 20523, German collection of microorganisms and tissue culture cells, Braunschweig, Germany), a strain often utilized for antimicrobial tests, was grown overnight at $37^{\circ} \mathrm{C}$ on Columbia sheep blood agar (BBL, $\mathrm{BD}$, Heidelberg, Germany). One inoculation loop of this culture was resuspended into $30 \mathrm{~mL}$ Brain Heart Infusion (BHI) (BD, BBL, Heidelberg, Germany) complemented with $1 \%$ sucrose [11]. The sterile titanium discs were positioned in 96-well microtiter plates (Techno Plastic Products AG, Trasadingen, Switzerland), covered with $100 \mu \mathrm{L}$ S. mutans suspension, and incubated aerobically at $37^{\circ} \mathrm{C}$. For S. mutans, we deliberately used no surface coating with salivary proteins, since this reduces the contact angle of titanium, and, consequently, adhesion of $S$. mutans would be reduced [12]. In preliminary tests we achieved the best biofilm forming results without a conditioning film. Every $24 \mathrm{~h} \mathrm{BHI}$ was changed. After $72 \mathrm{~h}$ the medium was drawn off, the discs were washed with $0.9 \% \mathrm{NaCl}$ solution and transferred into a new, sterile microtiter plate.

2.2. Multispecies Saliva Biofilm. To simulate a perimucositis situation, we used a multispecies saliva biofilm. Unstimulated saliva was taken from healthy donors $(n=5$, pooled saliva, mean age $29 \pm 7$ years, nonsmokers) as a source of oral microbiota. This was approved by the local ethics committee (BB 120/10). Saliva donors did not take any medication three months prior to the study and did not have active carious lesions or overt periodontal disease. In the saliva of three donors we found Aggregatibacter actinomycetemcomitans and Porphyromonas gingivalis, and Fusobacterium nucleatum in four donors and Streptococcus sanguinis in all five donors in a PCR analysis. These bacteria are typically associated with periodontitis, perimucositis, or peri-implantitis.

Sterile titanium discs were covered with $100 \mu \mathrm{L}$ saliva and incubated aerobically at $37^{\circ} \mathrm{C}$. We deliberately used no previous surface coating with salivary proteins, because we inoculated full saliva containing all proteins. The further procedure was as described above.
2.3. Multispecies Subgingival Biofilm. To study possible synergistic effects in an anaerobic milieu, we used a multispecies subgingival biofilm. Subgingival plaque was obtained from five periodontal patients (pooled plaque, mean age $50 \pm 10$ years). It was removed with a dental curet and resuspended in Schädler broth with vitamin $\mathrm{K} 1$ (BD, BBL, Heidelberg, Germany). The plaque was incubated at $37^{\circ} \mathrm{C}$ overnight under anaerobic conditions using the Anaerocult A system (Merck, Darmstadt, Germany) to develop a biofilm. Before biofilm formation the sterile titanium discs were conditioned with DTT saliva. To this end, $10 \mathrm{~mL}$ saliva was collected from healthy donors, pooled, and treated with $5 \mathrm{~mL} 1 \mathrm{M}$ DL-dithiothreitol solution (DTT, Sigma-Aldrich, Steinheim, Germany) and $5 \mathrm{~mL}$ distilled water. This DTT saliva mixture was then centrifuged (Mini Spin, Eppendorf, Hamburg Germany). Thereafter the supernatant was filtered through a $0.2 \mu \mathrm{m}$ pore filter (HVM Filtramed, Rotenburg an der Fulda, Germany) and frozen at $-20^{\circ} \mathrm{C}$ until use. The titanium discs were covered with $500 \mu \mathrm{L}$ DTT saliva and incubated $2 \mathrm{~h}$ at $37^{\circ} \mathrm{C}$. Afterwards the DTT saliva was carefully removed, and $1 \mathrm{~mL}$ plaque suspension was added and incubated anaerobically at $37^{\circ} \mathrm{C}$.

2.4. Antiseptic and Chemical Pretreatment. Titanium discs were pretreated with $0.1 \%$ chlorhexidine digluconate (CHX, aqueous solution, Fagron $\mathrm{GmbH} \&$ Co KG, Barsbüttel, Germany), $0.1 \%$ octenidine dihydrochloride (OCT, Schülke \& Mayr GmbH, Norderstedt, Germany), $0.1 \%$ polihexanide (PHMB, B. Braun, Melsungen, Germany), 0.6\% sodium hypochlorite ( $\mathrm{NaOCl}$, AppliChem $\mathrm{GmbH}$, Darmstadt, Germany), $1.5 \%$ hydrogen peroxide $\left(\mathrm{H}_{2} \mathrm{O}_{2}\right.$, AppliChem $\mathrm{GmbH}$, Darmstadt, Germany), and 20\% ethylenediaminetetraacetic acid (EDTA, AppliChem GmbH, Darmstadt, Germany). As negative control we used $0.9 \% \mathrm{NaCl}$ solution, hereafter called "NaCl control."

Biofilm discs were covered with $100 \mu \mathrm{L}$ of the substances and incubated for $30 \mathrm{~min}$. Within this incubation time, antiseptically pretreated biofilms were treated $1 \mathrm{~min}$ with plasma. Afterwards the antiseptic effect was halted by adding $1 \mathrm{~mL}$ inactivator. For inactivating PHMB effects we used $30 \mathrm{~g} / \mathrm{L}$ Tween $80,30 \mathrm{~g} / \mathrm{L}$ saponin, $1.0 \mathrm{~g} / \mathrm{L}$ histidin, and $1.0 \mathrm{~g} / \mathrm{L}$ cystein. For CHX, OCT, and EDTA, the inactivator was Lipofundin MCT 20\% (B. Braun, Melsungen, Germany). An inactivator consisting of $30 \mathrm{~g} / \mathrm{L}$ Tween $80,3 \mathrm{~g} / \mathrm{L}$ lecithin, $1 \mathrm{~g} / \mathrm{L}$ histidin and $5 \mathrm{~g} / \mathrm{L}$ sodium thiosulfate was used to stop the effect of $\mathrm{NaOCl}$ and $\mathrm{H}_{2} \mathrm{O}_{2}$. All inactivators were proven by the quantitative suspension test according to DIN EN 1040 (German Institute for Standardisation).

2.5. Plasma Treatment. To test the resistance of antiseptics against plasma treatment, we treated $5 \mathrm{~mL}$ of each antiseptic for 1 and $10 \mathrm{~min}$ with plasma. By spectral measurement (CHX: $231 \mathrm{~nm}$, OCT: $213 \mathrm{~nm}$, PHMB: $235 \mathrm{~nm}$ ) we compared measurement results after plasma treatment with untreated antiseptics.

For plasma generation we used the atmospheric pressure plasma jet kINPen 09 (neoplas tools, Greifswald, Germany) [8]. Argon (Ar) gas flow was set to $5 \mathrm{slm}$ (standard liters per 
minute). The flow rate was controlled by a flow controller (MKS Instruments, Munich, Germany). We had a constant pin-to-disc distance of $7 \mathrm{~mm}$ during the application. The kINPen was fixed in a computer driven 3 axes $(x, y, z)$ motorized stage, under which a 96-well plate with the titanium discs was positioned. The plasma devices were consecutively driven from well to well, positioned centrally over the discs and remained in position for $1 \mathrm{~min}$. To assess the effects of biofilm dehydration by gas flow, biofilms were treated with Ar gas without plasma generation. Additionally we investigated biofilms without gas or plasma treatment, hereafter called "control procedure."

After plasma treatment, titanium discs were placed into wells with $200 \mu \mathrm{L} 0.9 \% \mathrm{NaCl}$ solution and the biofilm was removed by ultrasonic scaling. Serial dilutions of the resuspended biofilm solution were made by transferring $0.1 \mathrm{~mL}$ of the resultant suspension to $0.9 \mathrm{~mL}$ of fresh $0.9 \%$ $\mathrm{NaCl}$ solution. Afterwards an aliquot portion of $0.1 \mathrm{~mL}$ from each dilution was plated on Columbia sheep blood agar (BBL, BD, Heidelberg, Germany) and incubated aerobically at $37^{\circ} \mathrm{C}$ for $48 \mathrm{~h}$ for $S$. mutans and saliva. Resuspended plaque biofilms were plated on Schädler agar (BBL, BD, Heidelberg, Germany) and incubated anaerobically at $37^{\circ} \mathrm{C}$ for $48 \mathrm{~h}$. The colonies were counted and expressed as colony forming units (CFU/mL).

2.6. Change in Treatment Order. In an additional experiment we changed the treatment order because we wanted to test the hypothesis that EDTA destroys the biofilm matrix to increase the plasma effect and that a higher antimicrobial effect after an increased radical induction of $\mathrm{H}_{2} \mathrm{O}_{2}$ by plasma treatment is observable. First saliva biofilms were treated with plasma, and then EDTA or $\mathrm{H}_{2} \mathrm{O}_{2}$ was added. After $30 \mathrm{~min}$ the antiseptic effect was halted by adding $1 \mathrm{~mL}$ inactivator as described above. Then, CFUs were determined.

2.7. Live-Dead Staining. Biofilms on discs were stained with fluorescein diacetate (FDA) and ethidium bromide (EB) to selectively stain living (green) and dead (red) bacteria [13]. Thereby $6 \mu \mathrm{L}$ of a FDA stock solution $(5 \mathrm{mg} / \mathrm{mL})$ and $3 \mu \mathrm{L}$ of an $\mathrm{EB}$ stock solution $(1.25 \mathrm{mg} / \mathrm{mL})$ were mixed in $1 \mathrm{~mL} 0.9 \%$ $\mathrm{NaCl}$ solution. The fluorescence was measured at excitation wavelengths of $485 \mathrm{~nm}$ and $530 \mathrm{~nm}$ and emission wavelengths of $530 \mathrm{~nm}$ and $630 \mathrm{~nm}$ on Berthold TriStar LB 941 (Berthold Technologies, Bad Wildbad, Germany). The division of the green fluorescent signal by the red fluorescent signal yielded the live-dead ratio. With the live-dead staining we tested the antimicrobial effect with all agents in both treatment orders on saliva biofilms. Additionally, samples were observed using a Zeiss CLSM510 Exciter confocal laser scanning microscope (Carl Zeiss MicroImaging GmbH, Jena, Germany). Using Live-dead staining the antimicrobial effect was tested with all agents in both treatment orders on saliva biofilms.

2.8. Statistics. For all analyses, observed CFU values were transferred to their base 10 logarithm (referred to as $\log _{10}$ CFU; for S. mutans: $\left.\log _{10}(\mathrm{CFU}+1)\right)$. Continuous data are presented as mean \pm standard deviation (SD).
The $\log _{10}$-reduction factor (RF) for each treatment method was calculated [14].

To examine differences in $\log _{10} \mathrm{CFU}$ values between different procedures and admixture combinations, $\log _{10} \mathrm{CFU}$ values were compared for each agent versus the $\mathrm{NaCl}$ control within each procedure (within columns of Table 1) and for each agent; differences between the $\mathrm{NaCl}$ control, Ar gas, and Ar plasma were pairwise evaluated (within rows of Table 1) using two-sided Mann-Whitney $U$ tests. To adjust for multiple testing within each step (comparisons within columns, resp., within rows), $P$ values were corrected according to Bonferroni.

Differences in $\log _{10} \mathrm{CFU}$ values across different procedures (control procedure, Ar gas, and Ar plasma) and agents $\left(\mathrm{NaCl}\right.$ control, $\mathrm{CHX}, \mathrm{OCT}, \mathrm{PHMB}, \mathrm{NaOCl}, \mathrm{H}_{2} \mathrm{O}_{2}$, and EDTA) were evaluated using linear regression analyses including the twofold interaction term of both factors (significant at $P<0.10$ ). Linear regression coefficients with their $95 \%$ confidence intervals (CI) were reported. $R^{2}$ quantifies the amount of variation explained by the model.

Differences of live-dead ratios across combinations of procedures and admixtures were evaluated using two-sided Mann-Whitney- $U$-tests. $P$ values were corrected according to Bonferroni.

Statistical differences were considered significant at a level alpha of $5 \%$. Statistical analyses were performed with STATA/SE 10.0 (Stata Corp LP, College Station, TX, USA) and R 2.13.0 (R Development Core Team, 2011).

\section{Results}

The measured spectral data of the antiseptics before and after plasma treatment were identical (OCT: 0.5, CHX: 1, PHMB: $1.1)$, indicating that the agents were not modified by plasma.

\section{1. $\log _{10} C F U$ Values after Administration of Agent Plus Plasma/Gas}

3.1.1. S. mutans Biofilm. The highest CFU reduction with no colonies being detected (values below detection limit) was achieved for six combinations (Table 1): $\mathrm{NaOCl}$ with any procedure and $\mathrm{H}_{2} \mathrm{O}_{2}$ with any procedure.

Compared with the control procedure, all agents lead to significantly lower CFU values compared with the negative control $(P<0.05)$. In combination with Ar gas, admixture of CHX $(\mathrm{RF}=1.96)$, OCT $(\mathrm{RF}=1.90)$, $\mathrm{PHMB}(\mathrm{RF}=3.39)$, $\mathrm{NaOCl}(\mathrm{RF}=6.91)$, and $\mathrm{H}_{2} \mathrm{O}_{2}(\mathrm{RF}=6.91)$ significantly reduced $\mathrm{CFU}$ values compared with $\mathrm{NaCl}$ controls. In combination with Ar plasma, admixture of PHMB $(\mathrm{RF}=3.36)$, $\mathrm{NaOCl}(\mathrm{RF}=6.91), \mathrm{H}_{2} \mathrm{O}_{2}(\mathrm{RF}=6.91)$, and EDTA $(\mathrm{RF}=$ 2.78) significantly reduced CFU values compared with $\mathrm{NaCl}$ controls.

Compared with the control procedure, only Ar plasma significantly reduced $\log _{10} \mathrm{CFU}$ values when combined with $\mathrm{NaCl}$ control, $\mathrm{PHMB}$, or EDTA $(P<0.05)$. For admixtures of $\mathrm{CHX}, \mathrm{OCT}, \mathrm{NaOCl}$, and $\mathrm{H}_{2} \mathrm{O}_{2}, \log _{10} \mathrm{CFU}$ values did not differ significantly across procedures. For $\mathrm{NaCl}$ control and 


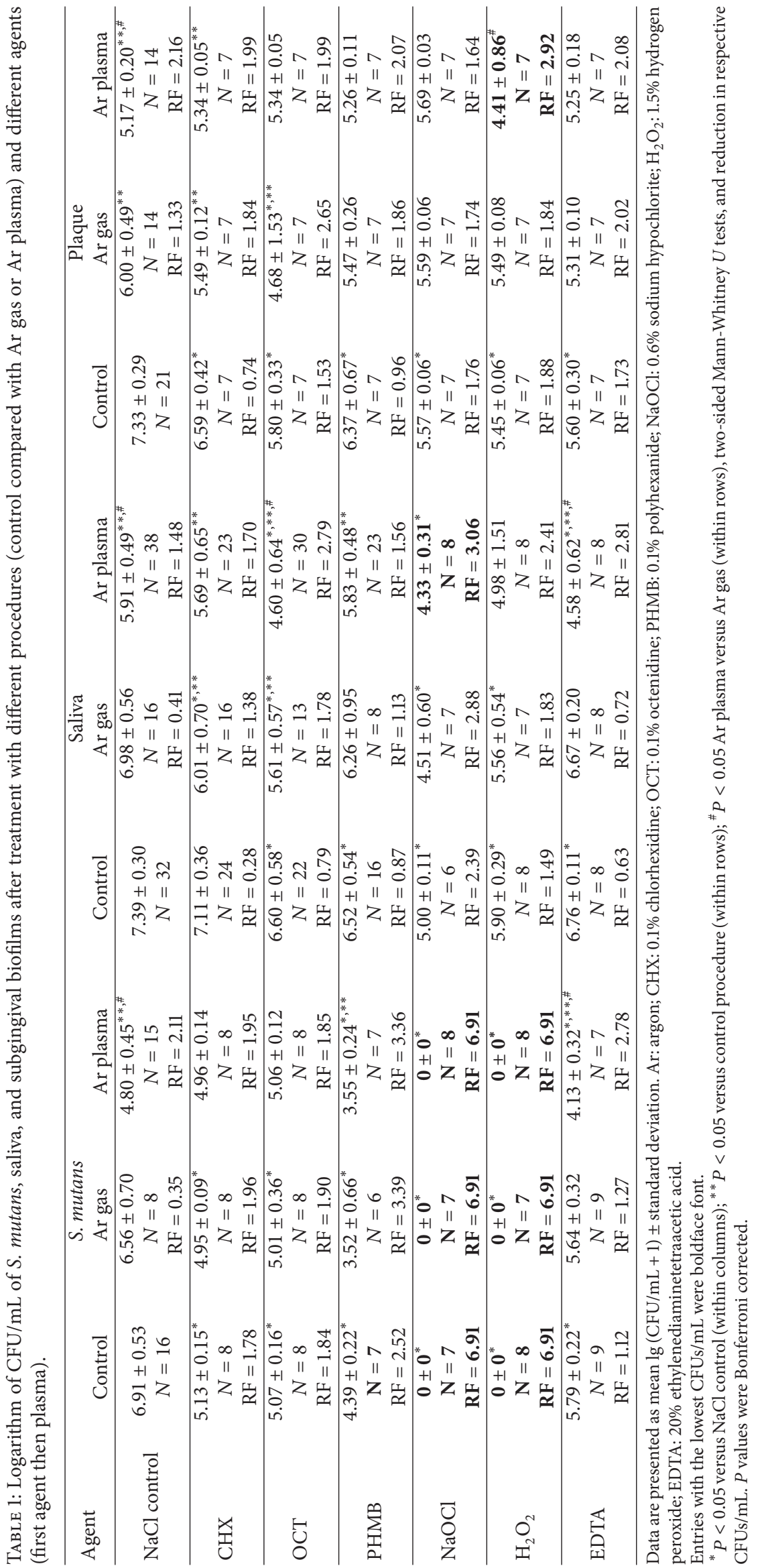


TABLE 2: Linear regression models evaluating effects of different agents and plasma procedures on $\lg (\mathrm{CFU} / \mathrm{mL}+1)$ for $S$. mutans, saliva, and subgingival biofilms (first agent then plasma).

\begin{tabular}{|c|c|c|c|c|c|c|}
\hline & \multicolumn{2}{|c|}{ S. mutans $\left(N=178, R^{2}=98.4 \%\right)$} & \multicolumn{2}{|c|}{ Saliva $\left(N=329, R^{2}=72.6 \%\right)$} & \multicolumn{2}{|c|}{ Subgingival $\left(N=175, R^{2}=76.3 \%\right)$} \\
\hline & $B(95 \% \mathrm{CI})$ & $P$ & $B(95 \% \mathrm{CI})$ & $P$ & $B(95 \% \mathrm{CI})$ & $P$ \\
\hline \multicolumn{7}{|l|}{ Agent (ref. $\mathrm{NaCl}$ control) } \\
\hline $\mathrm{CHX}$ & $-1.78(-2.06 ;-1.50)$ & $<0.001$ & $-0.29(-0.59 ; 0.02)$ & $<0.001$ & $-0.74(-1.12 ;-0.36)$ & $<0.001$ \\
\hline OCT & $-1.84(-2.12 ;-1.56)$ & $<0.001$ & $-0.79(-1.10 ;-0.49)$ & $<0.001$ & $-1.54(-1.91 ;-1.16)$ & $<0.001$ \\
\hline PHMB & $-2.51(-2.81 ;-2.22)$ & $<0.001$ & $-0.87(-1.22 ;-0.53)$ & $<0.001$ & $-0.96(-1.34 ;-0.58)$ & $<0.001$ \\
\hline $\mathrm{NaOCl}$ & $-6.91(-7.20 ;-6.61)$ & $<0.001$ & $-2.39(-2.89 ;-1.90)$ & $<0.001$ & $-1.76(-2.14 ;-1.38)$ & $<0.001$ \\
\hline $\mathrm{H}_{2} \mathrm{O}_{2}$ & $-6.91(-7.19 ;-6.63)$ & $<0.001$ & $-1.50(-1.94 ;-1.06)$ & $<0.001$ & $-1.89(-2.27 ;-1.51)$ & $<0.001$ \\
\hline EDTA & $-1.11(-1.38 ;-0.84)$ & $<0.001$ & $-0.64(-1.08 ;-0.19)$ & 0.005 & $-1.74(-2.12 ;-1.36)$ & $<0.001$ \\
\hline \multicolumn{7}{|l|}{ Procedure (ref. control) } \\
\hline Argas & $-0.35(-0.63 ;-0.07)$ & 0.02 & $-0.42(-0.76 ;-0.07)$ & 0.02 & $-1.34(-1.64 ;-1.04)$ & $<0.001$ \\
\hline Ar plasma & $-2.10(-2.34 ;-1.87)$ & $<0.001$ & $-1.49(-1.76 ;-1.22)$ & $<0.001$ & $-2.16(-2.46 ;-1.86)$ & $<0.001$ \\
\hline \multicolumn{7}{|c|}{ Interaction agent $\times$ procedure } \\
\hline $\mathrm{CHX} \times$ Ar gas & $0.18(-0.25 ; 0.60)$ & 0.42 & $-0.68(-1.17 ;-0.19)$ & 0.008 & $0.24(-0.31 ; 0.79)$ & 0.40 \\
\hline OCT $\times$ Ar gas & $0.29(-0.14 ; 0.71)$ & 0.19 & $-0.58(-1.10 ;-0.61)$ & 0.03 & $0.22(-0.33 ; 0.78)$ & 0.43 \\
\hline $\mathrm{PHMB} \times$ Ar gas & $-0.53(-0.99 ;-0.07)$ & 0.02 & $0.15(-0.44 ; 0.75)$ & 0.61 & $0.44(-0.12 ; 0.99)$ & 0.12 \\
\hline $\mathrm{NaOCl} \times$ Ar gas & $0.35(-0.10 ; 0.79)$ & 0.12 & $-0.07(-0.78 ; 0.64)$ & 0.84 & $1.35(0.80 ; 1.91)$ & $<0.001$ \\
\hline $\mathrm{H}_{2} \mathrm{O}_{2} \times$ Ar gas & $0.35(-0.09 ; 0.78)$ & 0.12 & $0.08(-0.59 ; 0.75)$ & 0.81 & $1.38(0.83 ; 1.94)$ & $<0.001$ \\
\hline EDTA $\times$ Ar gas & $0.19(-0.22 ; 0.61)$ & 0.36 & $0.32(-0.33 ; 0.98)$ & 0.33 & $1.05(0.50 ; 1.61)$ & $<0.001$ \\
\hline $\mathrm{CHX} \times$ Ar plasma & $1.93(1.53 ; 2.33)$ & $<0.001$ & $0.08(-0.34 ; 0.50)$ & 0.72 & $0.91(0.35 ; 1.46)$ & 0.001 \\
\hline OCT $\times$ Ar plasma & $2.09(1.70 ; 2.49)$ & $<0.001$ & $-0.51(-0.92 ;-0.10)$ & 0.02 & $1.70(1.15 ; 2.25)$ & $<0.001$ \\
\hline PHMB $\times$ Ar plasma & $1.26(0.85 ; 1.68)$ & $<0.001$ & $0.80(0.35 ; 1.25)$ & 0.001 & $1.04(0.49 ; 1.60)$ & $<0.001$ \\
\hline $\mathrm{NaOCl} \times$ Ar plasma & $2.10(1.70 ; 2.51)$ & $<0.001$ & $0.81(0.16 ; 1.47)$ & 0.02 & $2.27(1.72 ; 2.82)$ & $<0.001$ \\
\hline $\mathrm{H}_{2} \mathrm{O}_{2} \times$ Ar plasma & $2.10(1.70 ; 2.50)$ & $<0.001$ & $0.57(-0.05 ; 1.19)$ & 0.07 & $1.12(0.57 ; 1.67)$ & $<0.001$ \\
\hline EDTA $\times$ Ar plasma & $0.44(0.04 ; 0.84)$ & 0.03 & $-0.69(-1.31 ;-0.08)$ & 0.03 & $1.82(1.26 ; 2.37)$ & $<0.001$ \\
\hline
\end{tabular}

Ar: argon; CHX: 0.1\% chlorhexidine; OCT: 0.1\% octenidine; PHMB: 0.1\% polyhexanide; $\mathrm{NaOCl}: 0.6 \%$ sodium hypochlorite; $\mathrm{H}_{2} \mathrm{O}_{2}: 1.5 \%$ hydrogen peroxide; EDTA: 20\% ethylenediaminetetraacetic acid.

EDTA, $\log _{10}$ CFU values were significantly reduced for $\mathrm{Ar}$ plasma compared with Ar gas $(P<0.05)$.

To evaluate the impact of procedure and agents on $S$. mutans CFU values, linear regression models were evaluated (Table 2). The model explained $98.4 \%$ of the variation in observed CFU values. Procedure, admixture, and the interaction of both were significantly related to $\log _{10}(\mathrm{CFU} / \mathrm{mL})$ values $(P<0.001)$. Post hoc analysis confirmed that any combination with $\mathrm{NaOCl}$ or $\mathrm{H}_{2} \mathrm{O}_{2}$ was significantly the best performing combination among those tested $(P<0.001)$ with a predicted reduction of $7.51 \log _{10}(\mathrm{CFU} / \mathrm{mL})$ compared with $\mathrm{NaCl}$ control.

3.1.2. Saliva Biofilm. The highest reduction in $\log _{10} \mathrm{CFU}$ was found for the combination of Ar plasma with $\mathrm{NaOCl}$ $(\mathrm{RF}=3.06$, Table 1$)$. Compared with the control procedure, admixture of OCT, PHMB, $\mathrm{NaOCl}, \mathrm{H}_{2} \mathrm{O}_{2}$, and EDTA led to significantly lower CFU values compared with the $\mathrm{NaCl}$ control $(P<0.05)$. Combined with Ar gas, admixture of CHX, OCT, $\mathrm{NaOCl}$, and $\mathrm{H}_{2} \mathrm{O}_{2}$ significantly reduced CFUs compared with $\mathrm{NaCl}$ control $(P<0.05)$. The effect of $\mathrm{Ar}$ plasma on $\mathrm{CFU}$ reduction was significantly enhanced by OCT, $\mathrm{NaOCl}$, and EDTA compared with the $\mathrm{NaCl}$ control.

Compared with the control procedure, application of Ar gas significantly reduced CFU values when $\mathrm{CHX}$ or
OCT was applied previously $(P<0.05)$. Compared with the control procedure, Ar plasma treatment significantly enhanced antimicrobial effects of $\mathrm{NaCl}$ control, CHX, OCT, PHMB, and EDTA $(P<0.05)$. For $\mathrm{NaCl}$ control, OCT and EDTA, Ar plasma performed significantly better compared with Ar gas $(P<0.05)$.

For the combination of plasma and agents, the simple additive effect on $\log _{10}$ CFU reduction was partly exceeded. Compared with single reduction factors for plasma and CHX $(\mathrm{RF}=1.48$ and $\mathrm{RF}=0.28$, resp. $)$, the combined $\mathrm{RF}$ was 1.70 (Table 1).

Estimating the impact of procedure and agents on saliva $\log _{10}$ CFU values more closely (Table 2 ), linear regression models were performed, which explained $72.5 \%$ of the variation in observed CFU values. Procedure, admixture, and the interaction of both were significantly related to $\log _{10}$ CFU values $(P<0.001)$. Post hoc analyses revealed that the combination of Ar plasma with $\mathrm{NaOCl}$ was the best performing combination among those tested with a predicted reduction of $3.07 \log _{10}$ CFU compared with the total negative control ( $\mathrm{NaCl}$ control with control procedure). It was significantly better compared with most combinations but similarly effective compared with Ar gas combined with $\mathrm{NaOCl}(P=0.52)$, Ar plasma combined with OCT $(P=$ $0.22)$, and Ar plasma combined with EDTA $(P=0.38)$. 
3.1.3. Subgingival Biofilm. The highest $\log _{10} \mathrm{CFU}$ reduction was found for Ar plasma with $\mathrm{H}_{2} \mathrm{O}_{2}(4.41 \pm 0.86$, Table 1). For the control procedure any admixture led to significantly reduced $\log _{10} \mathrm{CFU}$ values compared with $\mathrm{NaCl}$ control $(P<$ 0.05). With Ar gas, only the admixture of OCT was beneficial $\left(\log _{10} \mathrm{CFU} 4.68 \pm 1.53\right)$. Regardless of the admixture, combinations with Ar plasma led to comparable reductions in $\log _{10}$ CFU values.

Considering the effect of the three procedures within agents, both Ar gas and Ar plasma significantly reduced $\log _{10} \mathrm{CFU}$ values compared with the control procedure for $\mathrm{NaCl}$ control and $\mathrm{H}_{2} \mathrm{O}_{2}$. For $\mathrm{PHMB}, \mathrm{NaOCl}$, and EDTA, $\log _{10} \mathrm{CFU}$ values did not differ significantly across procedures. For $\mathrm{NaCl}$ control and $\mathrm{H}_{2} \mathrm{O}_{2}, \log _{10} \mathrm{CFU}$ values were significantly reduced for Ar plasma compared with Ar gas. The overall best $\log _{10} \mathrm{CFU}$ reduction was achieved when $\mathrm{Ar}$ plasma was combined with $\mathrm{H}_{2} \mathrm{O}_{2}(\mathrm{RF}=2.92)$.

To evaluate the impact of procedure and agents on plaque $\log _{10} \mathrm{CFU}$ values, linear regression models were evaluated (Table 2). While $76.3 \%$ of the variation in observed $\log _{10} \mathrm{CFU}$ values were explained, procedure, admixture, and the interaction of both were significantly related to $\log _{10} \mathrm{CFU}$ values $(P<$ 0.001). Post hoc analyses revealed that the combination of Ar plasma with $\mathrm{H}_{2} \mathrm{O}_{2}$ was significantly the best performing combination among those tested $(P<0.001)$ with the exception of Ar gas with OCT $(P=0.25)$. The combination of Ar plasma with $\mathrm{H}_{2} \mathrm{O}_{2}$ had a predicted reduction of 2.92 $\log _{10} \mathrm{CFU}$ compared with the total negative control $(\mathrm{NaCl}$ control with control procedure).

\section{2. $\log _{10} C F U$ Values after Administration of Plasma Plus} Agent. To evaluate whether the reduction in CFU was related to the procedure or the admixture, the treatment order was changed; that is, plasma or gas was applied before the admixture of agents (Table 3 ). The data were comparable with those of Table 1 . The extent of $\log _{10} \mathrm{CFU}$ reduction relative to the total negative control was comparable, irrespective of the treatment order. For example, the combination of EDTA plus Ar plasma achieved a $\log _{10} \mathrm{CFU}$ reduction of $\mathrm{RF}=2.81$, while the combination of both in reversed treatment order achieved a $\log _{10}$ CFU reduction of $\mathrm{RF}=2.66$. For the combination of $\mathrm{H}_{2} \mathrm{O}_{2}$ with Ar plasma, respective $\log _{10} \mathrm{CFU}$ reductions were 2.41 and 2.58. Accordingly, we found an additive effect of the antimicrobial effects of plasma $\left(\mathrm{RF}_{\text {plasma }}=1.35\right)$ and $\mathrm{H}_{2} \mathrm{O}_{2}\left(\mathrm{RF}_{\mathrm{H}_{2} \mathrm{O}_{2}}=1.00\right)$ when combined: $\mathrm{RF}_{\text {plasma }+\mathrm{H}_{2} \mathrm{O}_{2}}=2.58$ $(>1.35+1.00)$. The same figures applied to the combination of plasma $\left(\mathrm{RF}_{\text {plasma }}=1.35\right)$ with EDTA $\left(\mathrm{RF}_{\mathrm{EDTA}}=1.25\right)$ and plasma + EDTA: $\mathrm{RF}_{\text {plasma+EDTA }}=2.66(\sim 1.35+1.25)$.

3.3. Live-Dead Staining. To determine the antimicrobial effect of all combinations with both treatment orders, we stained the biofilms with EB and FDA (Figure 1). A reduction of the living microorganisms in \% can be explained by a reduced FDA fluorescence or an increased EB fluorescence signal, resulting in a higher number of dead cells. The negative control achieved a mean value of $100 \%$. For Ar plasma treated cells the value decreased to $45.3 \%$. After EDTA treatment the mean living microorganisms of $56.4 \%$ were further reduced
TABLE 3: Logarithm of saliva biofilm CFU/mL after treatment with different procedures (control compared with Ar gas or Ar plasma) and different agents $\left(\mathrm{NaCl}, \mathrm{H}_{2} \mathrm{O}_{2}\right.$, and EDTA) in changed order (first plasma then agent treatment).

\begin{tabular}{lccc}
\hline \multirow{2}{*}{ Agent } & \multicolumn{3}{c}{ Procedure } \\
& Control & Ar gas & Ar plasma \\
\hline \multirow{2}{*}{$\mathrm{NaCl}$ control } & $7.17 \pm 0.48$ & $6.59 \pm 0.48^{* *}$ & $5.82 \pm 0.52^{* *, \#}$ \\
& $N=21$ & $N=15$ & $N=8$ \\
$\mathrm{H}_{2} \mathrm{O}_{2}$ & $6.17 \pm 0.80^{*}$ & $5.62 \pm 0.86^{*}$ & $4.59 \pm 0.77^{*, * *, \#}$ \\
& $N=14$ & $N=15$ & $N=11$ \\
EDTA & $5.92 \pm 0.37^{*}$ & $6.25 \pm 0.43^{* *}$ & $4.51 \pm 0.82^{*, * *, \#}$ \\
& $N=8$ & $N=14$ & $N=16$ \\
\hline
\end{tabular}

Data are presented as mean $\lg (\mathrm{CFU} / \mathrm{mL}+1) \pm \mathrm{SD}$.

Ar: argon; CHX: 0.1\% chlorhexidine; OCT: 0.1\% octenidine; PHMB: 0.1\% polyhexanide; $\mathrm{NaOCl}$ : $0.6 \%$ sodium hypochlorite; $\mathrm{H}_{2} \mathrm{O}_{2}: 1.5 \%$ hydrogen peroxide; EDTA: $20 \%$ ethylenediaminetetraacetic acid.

${ }^{*} P<0.05$ versus $\mathrm{NaCl}$ control (within columns); ${ }^{* *} P<0.05$ versus control procedure (within rows); ${ }^{\#} P<0.05$ comparing Ar plasma versus Ar gas (within rows), two-sided Mann-Whitney $U$ test, and reduction in saliva $\mathrm{CFU} / \mathrm{mL}$. $P$ values were Bonferroni corrected.

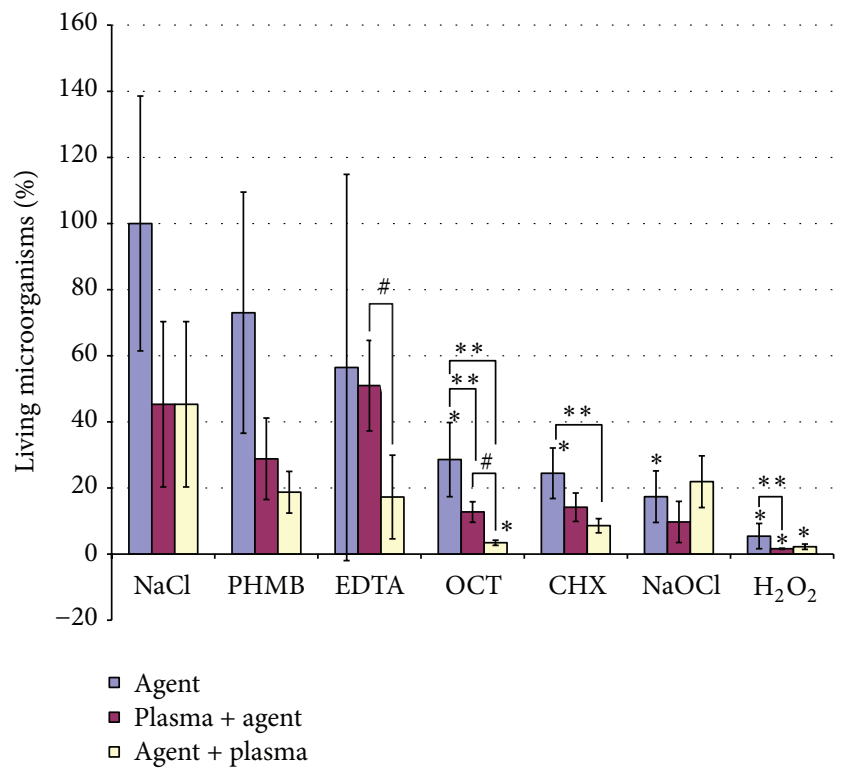

FIGURE 1: Green fluorescence (fluoresceindiacetate (FDA); alive): red fluorescence (ethidiumbromide (EB); damaged membrane dead) ratio of saliva biofilms after treatment with different procedures. Data are presented as mean $\pm \mathrm{SD}(n=8) .{ }^{*} P<0.05$ versus $\mathrm{NaCl}$ control (within equally colored columns); ${ }^{* *} P<0.05$ versus Agent only for each agent separately; ${ }^{\#} P<0.05$ Plasma plus Agent versus Agent plus plasma for each agent separately. $P$ values were retrieved from Mann-Whitney- $U$ tests and were Bonferroni corrected.

to $17.3 \%$ after additional plasma treatment. When EDTA treatment was applied after Ar plasma treatment, no additive effect was found (51\%). Both values differed significantly between the two treatment orders $(P<0.05)$.

Concerning antiseptics, additional Ar plasma treatment consistently decreased the mean ratio with only minimal differences between either treatment order (CHX: 24.4\%, 
CHX + Ar plasma: 8.6\%, and Ar plasma + CHX: 14.2\%; OCT: $28.6 \%$, OCT + Ar plasma: 3.4\%; Ar plasma + OCT: 14.17; PHMB: 73\%; PHMB + Ar plasma: $18.7 \%$, Ar plasma + PHMB: 28.8\%). Applying the antiseptic treatment before Ar plasma treatment led to slightly fewer living microorganisms compared with the other treatment order. This difference was significant $(P<0.05)$ for OCT. For $\mathrm{NaOCl}$, the lowest ratio was achieved when plasma treatment was applied prior to $\mathrm{NaOCl}$ treatment $(17.4 \% ; 21.9 \%$ when Ar plasma treatment was applied after $\mathrm{NaOCl}$ treatment). The highest reduction was achieved by $\mathrm{H}_{2} \mathrm{O}_{2}$ (5.4\%). With prior (posterior) Ar plasma treatment the ratio was $1.6 \%(2.2 \%)$.

Using confocal laser scanning microscopy (Figure 2) the difference between the $\mathrm{H}_{2} \mathrm{O}_{2}$ treatments was more pronounced in the center of the disc, where no green fluorescence was visible. The micrographs for both treatment orders for the combination of EDTA and Ar plasma were comparable. Using the same microscopic settings the micrographs of Ar plasma and antiseptic after treated biofilms were comparable to those of EDTA and Ar plasma. Therefore the pictures have not been presented here.

\section{Discussion}

In this study we found an additive antimicrobial effect between Ar plasma and agents irrespective of the treatment order regarding cultivation. Concerning Live-dead staining we found different antimicrobial effects dependent on treatment order.

Decontamination of dental implants is a promising application of plasma devices. To investigate synergistic effects between Ar plasma and agents, we used three different biofilm models.

In this study, sensitivity to treatments differed significantly between our biofilm models. Monospecies S. mutans biofilms were more susceptible to antimicrobial treatments than our multispecies biofilm models. S. mutans biofilm is no satisfactory model to test antibiofilm efficacy, because it cannot mimic oral conditions in an adequate way. Therefore we used biofilms cultured from ex vivo saliva and subgingival dental plaque to test Ar plasma in more realistic models. It was shown that Gram-negative as well as anaerobic bacteria are more sensitive to plasma than Gram positives or aerobic ones [15]. In multispecies saliva and subgingival biofilms we found both Gram-negative and -positive bacteria. We detected the highest CFU reduction by Ar plasma for the subgingival plaque biofilm. However, during biofilm processing we could not maintain the anaerobic atmosphere, which may have distorted the antimicrobial effect of the treatments.

Using only culture-based techniques to determine antimicrobial effects bears some disadvantages. Bacteria can exist in a viable but nonculturable state (VBNC) [16]. VBNC bacteria cannot be detected using culture-based techniques. Additionally, not all species in our multispecies biofilm grow on sheep blood agar. Therefore we used Live-dead staining with a microscopic and a spectroscopic analysis in saliva biofilms. This method showed more differences between treatment regimes.
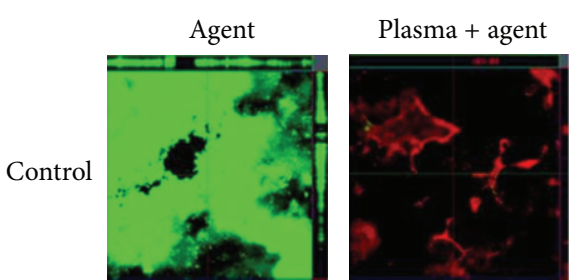

Agent + plasma
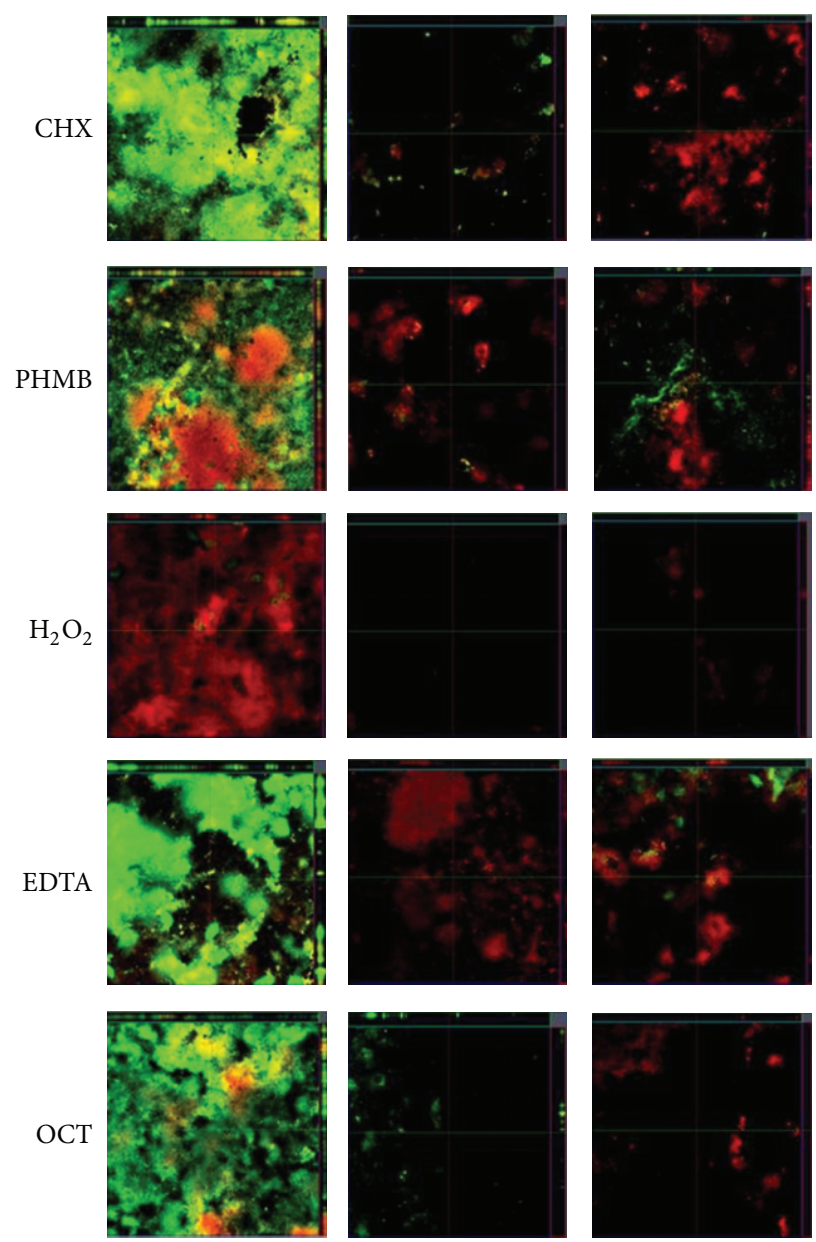

FIGURE 2: Fluorescence staining ofsaliva biofilms on titanium discs for different treatments: EDTA, EDTA plus Ar Plasma, Ar Plasma plus EDTA, $\mathrm{H}_{2} \mathrm{O}_{2}, \mathrm{H}_{2} \mathrm{O}_{2}$ plus Ar plasma and Ar plasma plus $\mathrm{H}_{2} \mathrm{O}_{2}$. The biofilms were stained with fluoresceindiacetate (FDA) and ethidiumbromide (EB) to selectively stain living (green) and dead (red) bacteria.

In this study, we found additive effects between Ar plasma and agents. To understand the magnitude of these effects, we compared the CFU and live-dead ratio reduction of $\mathrm{Ar}$ plasma and the agents and the CFU and live-dead-ratio reduction of the combination of both.

Concerning culture-based technique, the antibiofilm effect of Ar plasma was significantly increased for S. mutans biofilms by adding EDTA. No colonies were detectable after $\mathrm{NaOCl}$ or $\mathrm{H}_{2} \mathrm{O}_{2}$ treatment. With anaerobic multispecies biofilms we found significant synergistic effects between $\mathrm{H}_{2} \mathrm{O}_{2}$ and Ar plasma, which was also the procedure with the highest $\log _{10} \mathrm{CFU}$ reduction of saliva biofilm. For aerobic 
multispecies saliva biofilms significant additive effects between Ar plasma and EDTA and OCT were found. $\log _{10}$ CFU reductions for combined treatments were always higher than single Ar plasma or single agent treatments.

Treatment order differed significantly between addition of EDTA and octenidine before or after plasma application $(P<0.05)$ when we evaluated the results with Live-dead staining. When EDTA treatment was applied after Ar plasma treatment, no additive effect was found. Concerning antiseptics, additional Ar plasma treatment consistently decreased the mean ratio irrespective of the treatment order. For $\mathrm{NaOCl}$, the lowest ratio was achieved when plasma treatment was applied prior to $\mathrm{NaOCl}$ treatment. The highest reduction was achieved by $\mathrm{H}_{2} \mathrm{O}_{2}$.

To our knowledge there are no studies that have investigated the combined effects of agents and plasma. To understand our observed results, we limit the discussion to studies that combined the agents with UV, ozone, and radicals. According to different mechanisms, agents used in this study can be classified into four groups: antiseptics (CHX, $\mathrm{PHMB}$, and $\mathrm{OCT}$ ), $\mathrm{NaOCl}$, EDTA, and $\mathrm{H}_{2} \mathrm{O}_{2}$. With all agents, plasma interacts by the three main plasma constituents: UV radiation, ozone, and radicals. For each agent different mechanisms may be responsible for the increased effectivity of the combination of agent and plasma.

Firstly, no information has been published about interactions between radicals or radiation and antiseptics (CHX, PHMB, and OCT). After enquiry, the manufacturers confirmed UV stability of the products. Our spectral measurement of the antiseptics exposed before and after plasma treatment affirmed their information: antiseptics were not destroyed by the plasma treatment regime in our experiments. The antimicrobial efficacy of antiseptics was not decreased by additional plasma treatment as measured by CFU. For saliva biofilms the combination exceeded the pure plasma effect, which was corroborated by the live-dead staining experiment. Both treatment orders increased the antimicrobial effect, while prior plasma treatment tended to somewhat stronger antimicrobial effects. For OCT there was a significant difference between the two treatment orders: the biofilm could be destroyed by plasma so that the antiseptic could be more effective. Therefore, we might conclude that plasma does not destroy the antiseptics but rather has an additive effect in antiseptic treatment. For future medical therapies the combination of antiseptics and plasma treatment might be beneficial.

It was shown that the combination of $\mathrm{UV}$ and $\mathrm{NaOCl}$ increased the inactivation of some phages, but without any synergistic effects concerning E.coli and enterococci in waste water [17]. The synergistic effect between hypochlorite and radicals increases protein damage [18], which can be relevant for the increase in antimicrobial effects. Because of the high antimicrobial efficacy of $\mathrm{NaOCl}$ in single treatment, combinations with Ar plasma lead to comparable $\log _{10}$ CFUs for saliva biofilm $(P>0.05)$. Nevertheless, the combination with Ar plasma resulted in the highest CFU reduction factor, which was confirmed with Livedead staining. The antimicrobial effect was not increased for $\mathrm{NaOCl}$ plus Ar plasma but for Ar plasma plus $\mathrm{NaOCl}$.
Regarding S. mutans biofilms and plaque our results are consistent.

Thirdly, the antibiofilm effect of EDTA is based on the dispersal of the biofilm structure [19]. Our working hypothesis was that after disruption of the biofilm matrix antimicrobial agents like plasma can kill the bacteria directly without being inhibited by the protective matrix. This explains why antibiotics can act more effectively with EDTA [20]. UV in combination with ozone or $\mathrm{H}_{2} \mathrm{O}_{2}$ is necessary for EDTA degradation, whereas pure ozone did not alter the action EDTA [21]. In plasma processes UV and ozone are developed. The resulting $\mathrm{OH}$ radicals degrade EDTA without creating toxic degradation products [22]. It is possible that EDTA was destroyed by plasma and the main effect of this combination is the dispersion of the biofilms by EDTA. EDTA showed per se an antimicrobial effect which could be increased using plasma. For S. mutans und saliva, Ar plasma plus EDTA was significantly more effective than Ar plasma or EDTA alone. According to our hypothesis, this synergistic effect is a consequence of the biofilm dispersion and the resulting better efficacy of plasma. To confirm this hypothesis, we changed the treatment order: first plasma, then EDTA. This order resulted in the same efficacy as that using CFU analysis. Using Live-dead staining we found only minimal differences between EDTA and plasma plus EDTA but a higher reduction of the live-dead ratio applying EDTA plus plasma $(P<$ 0.05). This confirmed our hypothesis in agreement with other research groups who have combined EDTA with antibiotics [20].

Fourthly, the synergistic $\mathrm{UV}-\mathrm{H}_{2} \mathrm{O}_{2}$ effect is already known and is used to disinfect food packaging materials [23] and to clean waste water [24]. The mechanism is based on the photolysis of $\mathrm{H}_{2} \mathrm{O}_{2}$. The reaction is induced by the absorption of photons by hydrogen peroxide, which leads to the production of $\mathrm{OH}$ radicals [25]. According to our hypothesis the additionally formed radicals support antimicrobial effects of $\mathrm{H}_{2} \mathrm{O}_{2}$ and plasma. To test this hypothesis, we switched the treatment order of $\mathrm{H}_{2} \mathrm{O}_{2}$ and plasma too. We found the same synergistic reduction effect after switching the order using determination of CFU and Live-dead staining, whereas a prior $\mathrm{H}_{2} \mathrm{O}_{2}$ treatment tended to increase antimicrobial effects.

The antimicrobial effect of plasma may be attributed to a complex interaction of its components, for example, UV, radicals, ozone, and so forth. However, so far only single components such as UV have been combined with agents $[18,23,26]$. The current results deliver further information to understand antimicrobial effects of plasma in combination with agents as compared with effects of combinations between UV and agents because here a mixture of UV, radicals, and so forth was effective.

There are some studies which determined the cytotoxic potential of single treatments with plasma and agents [2729]. The toxic potential of the combination of plasma and agents needs further clarification. More investigations are necessary to identify the underlying mechanisms.

We performed in vitro studies. However, in vivo studies are necessary to ensure that (i) antimicrobial effects are not restricted to in vitro settings and (ii) application of combined 
treatments with Ar plasma and agents in clinical settings is reasonable.

This is the first study evaluating the combined antimicrobial effect of plasma with different agents using three different biofilm models. The combination of plasma and agents increased the antimicrobial efficacy of all tested compounds. It supports the additional use of plasma treatment of dental implants in addition to the often used chemicals and antiseptic solutions. Thus, the combined treatment with plasma and agents seems very beneficial, since the efficacy will be increased, while no additional chemicals will be needed. Furthermore, our results indicate that even a reduction of commonly used agents could be possible when applied in combination with Ar plasma, which could reduce the costs for treatments as well as the possible risk for patients due to the antimicrobial agents.

\section{Acknowledgments}

This work was realized within the framework of the multidisciplinary research cooperation "Campus PlasmaMed", particularly within the project "PlasmaDent." The authors acknowledge that this work was supported by a grant funded by the German Ministry of Education and Research (BMBF, Grant no. 13N9779). All titanium discs were kindly provided by Straumann (Institut Straumann AG, Basel, Switzerland). The authors thank Claudia Lehnert for her excellent technical assistance, Rüdiger Titze for his skillful support in operating the plasma equipment, and Peter Meisel for critical reading of the paper.

\section{References}

[1] G. E. Morfill, M. G. Kong, and J. L. Zimmermann, "Focus on plasma medicine," New Journal of Physics, vol. 11, no. 11, Article ID 115011, 2009.

[2] E. Stoffels, A. J. Flikweert, W. W. Stoffels, and G. M. W. Kroesen, "Plasma needle: a non-destructive atmospheric plasma source for fine surface treatment of (bio)materials," Plasma Sources Science and Technology, vol. 11, no. 4, pp. 383-388, 2002.

[3] A. Kramer, O. Assadian, H. Below et al., "Perspektiven der Plasmamedizin," Vakuum in Forschung Und Praxis, vol. 22, no. 2, pp. 33-38, 2010.

[4] I. Koban, K. Duske, L. Jablonowski et al., "Atmospheric plasma enhances wettability and osteoblast spreading on dentin in vitro: proof-of-principle," Plasma Processes and Polymers, vol. 8, no. 10, pp. 975-982, 2011.

[5] I. Koban, B. Holtfreter, N.-O. Hübner et al., "Antimicrobial efficacy of non-thermal plasma in comparison to chlorhexidine against dental biofilms on titanium discs in vitro-proof of principle experiment," Journal of Clinical Periodontology, vol. 38, no. 10, pp. 956-965, 2011.

[6] I. Koban, R. Matthes, N.-O. Hübner et al., "Treatment of Candida albicans biofilms with low-temperature plasma induced by dielectric barrier discharge and atmospheric pressure plasma jet," New Journal of Physics, vol. 12, Article ID 073039, 2010.

[7] G. Fridman, G. Friedman, A. Gutsol, A. B. Shekhter, V. N. Vasilets, and A. Fridman, "Applied plasma medicine," Plasma Processes and Polymers, vol. 5, no. 6, pp. 503-533, 2008.
[8] K.-D. Weltmann, E. Kindel, R. Brandenburg et al., "Atmospheric pressure plasma jet for medical therapy: plasma parameters and risk estimation," Contributions to Plasma Physics, vol. 49, no. 9, pp. 631-640, 2009.

[9] H. Halfmann, N. Bibinov, J. Wunderlich, and P. Awakowicz, "A double inductively coupled plasma for sterilization of medical devices," Journal of Physics D, vol. 40, no. 14, pp. 4145-4154, 2007.

[10] H. Halfmann, B. Denis, N. Bibinov, J. Wunderlich, and P. Awakowicz, "Identification of the most efficient VUV/UV radiation for plasma based inactivation of Bacillus atrophaeus spores," Journal of Physics D, vol. 40, no. 19, pp. 5907-5911, 2007.

[11] J. Merritt, F. Qi, S. D. Goodman, M. H. Anderson, and W. Shi, "Mutation of luxS affects biofilm formation in Streptococcus mutans," Infection and Immunity, vol. 71, no. 4, pp. 1972-1979, 2003.

[12] Y. Fujioka-Hirai, Y. Akagawa, and S. Minagi, "Adherence of Streptococcus mutans to implant materials," Journal of Biomedical Materials Research, vol. 21, no. 7, pp. 913-920, 1987.

[13] L. Netuschil, M. Brecx, K. G. Vohrer, and P. Riethe, "Vital fluorescence to assess in vitro and in vivo the antibacterial effects of amalgams," Acta stomatologica Belgica, vol. 93, no. 3, pp. 129-134, 1996.

[14] G. Müller, Y. Winkler, and A. Kramer, "Antibacterial activity and endotoxin-binding capacity of Actisorb Silver 220," Journal of Hospital Infection, vol. 53, no. 3, pp. 211-214, 2003.

[15] S. K. Filoche, C. H. Sissons, R. E. J. Sladek, and E. Stoffels, "Cold plasma treatment of in vitro dental plaque," in Plasma Assisted Decontamination of Biological and Chemical Agents, S. Güceri and A. Fridman,, Eds., pp. 65-78, Springer, Dordrecht, The Netherlands, 2008.

[16] G. Bogosian and E. V. Bourneuf, "A matter of bacterial life and death," EMBO Reports, vol. 2, no. 9, pp. 770-774, 2001.

[17] M. Montemayor, A. Costan, F. Lucena et al., "The combined performance of UV light and chlorine during reclaimed water disinfection," Water Science and Technology, vol. 57, no. 6, pp. 935-940, 2008.

[18] C. L. Hawkins, M. D. Rees, and M. J. Davies, "Superoxide radicals can act synergistically with hypochlorite to induce damage to proteins," FEBS Letters, vol. 510, no. 1-2, pp. 41-44, 2002.

[19] E. Banin, K. M. Brady, and E. P. Greenberg, "Chelator-induced dispersal and killing of Pseudomonas aeruginosa cells in a biofilm," Applied and Environmental Microbiology, vol. 72, no. 3, pp. 2064-2069, 2006.

[20] I. Raad, I. Chatzinikolaou, G. Chaiban et al., "In vitro and ex vivo activities of minocycline and EDTA against microorganisms embedded in biofilm on catheter surfaces," Antimicrobial Agents and Chemotherapy, vol. 47, no. 11, pp. 3580-3585, 2003.

[21] J. B. Rodríguez, A. Mutis, M. C. Yeber, J. Freer, J. Baeza, and H. D. Mansilla, "Chemical degradation of EDTA and DTPA in a totally chlorine free (TCF) effluent," Water Science and Technology, vol. 40, no. 11-12, pp. 267-272, 1999.

[22] M. Sorensen, S. Zurell, and F. H. Frimmel, "Degradation pathway of the photochemical oxidation of ethylenediaminetetraacetate (EDTA) in the UV/H2O2-process," Acta Hydrochimica et Hydrobiologica, vol. 26, no. 2, pp. 109-115, 1998.

[23] J. S. Reidmiller, J. D. Baldeck, G. C. Rutherford, and R. E. Marquis, "Characterization of UV-peroxide killing of bacterial spores," Journal of Food Protection, vol. 66, no. 7, pp. 1233-1240, 2003. 
[24] P. B. L. Chang and T. M. Young, "Kinetics of methyl tertbutyl ether degradation and by-product formation during UV/hydrogen peroxide water treatment," Water Research, vol. 34, no. 8, pp. 2233-2240, 2000.

[25] O. Johansson, J. Bood, M. Aldén, and U. Lindblad, "Hydroxyl radical consumption following photolysis of vapor-phase hydrogen peroxide at $266 \mathrm{~nm}$ : Implications for photofragmentation laser-induced fluorescence measurements of hydrogen peroxide," Applied Physics B, vol. 97, no. 2, pp. 515-522, 2009.

[26] Q.-F. Zeng, J. Fu, Y.-T. Shi, D.-S. Xia, and H.-L. Zhu, "Adsorbable organic halogens generation and reduction during degradation of phenol by UV radiation/sodium hypochlorite," Water Environment Research, vol. 81, no. 2, pp. 178-183, 2009.

[27] C. Bender, R. Matthes, E. Kindel et al., "The irritation potential of nonthermal atmospheric pressure plasma in the HET-CAM," Plasma Processes and Polymers, vol. 7, no. 3-4, pp. 318-320, 2010.

[28] G. Müller and A. Kramer, "Biocompatibility index of antiseptic agents by parallel assessment of antimicrobial activity and cellular cytotoxicity," Journal of Antimicrobial Chemotherapy, vol. 61, no. 6, pp. 1281-1287, 2008.

[29] G. Müller, A. Kramer, and J. Siebert, "Effectiveness of octenidine and chlorhexidine in the artificially contaminated 3-D-culture of human keratinocytes," GMS Krankenhaushyg Interdiszip, vol. 4, no. 2, p. Doc14, 2009 


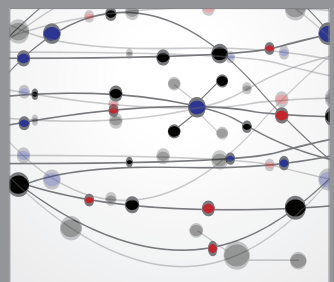

The Scientific World Journal
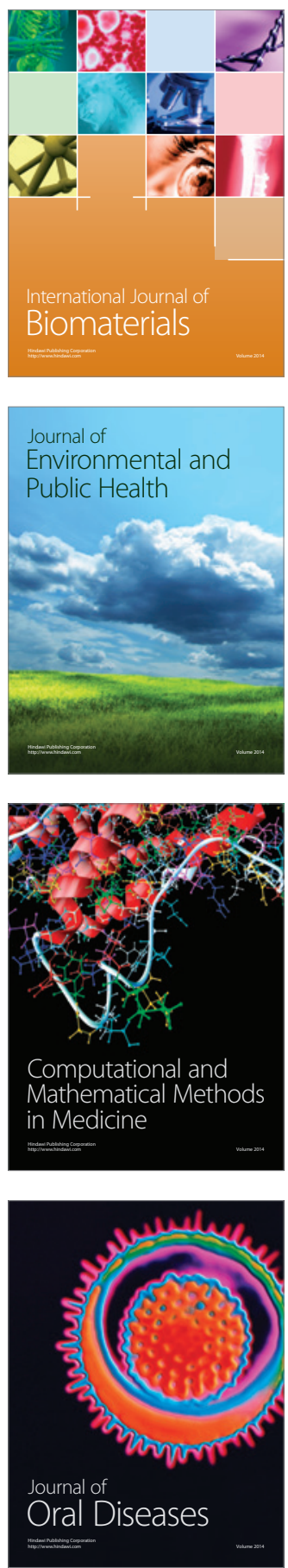
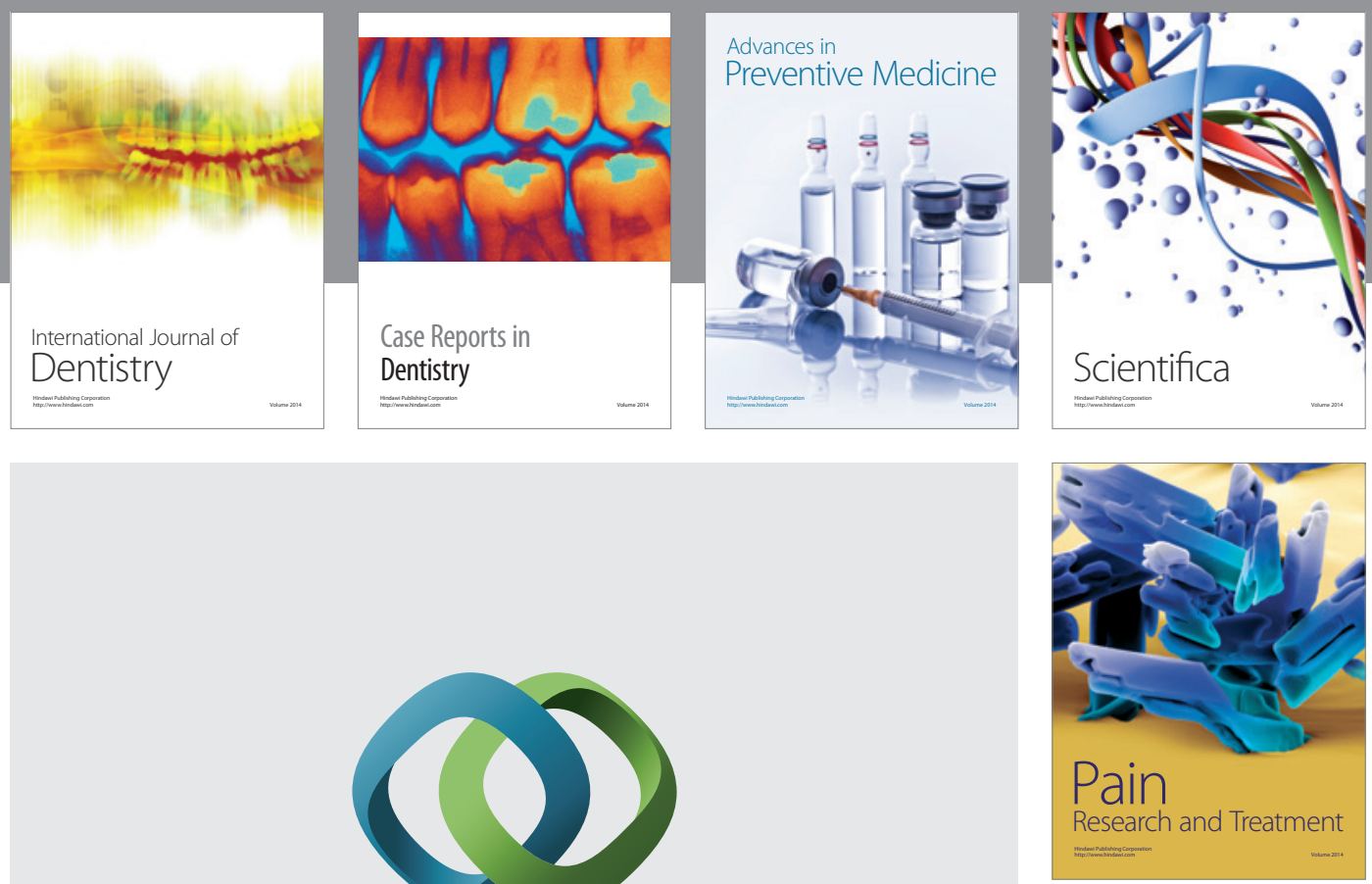

\section{Hindawi}

Submit your manuscripts at

http://www.hindawi.com
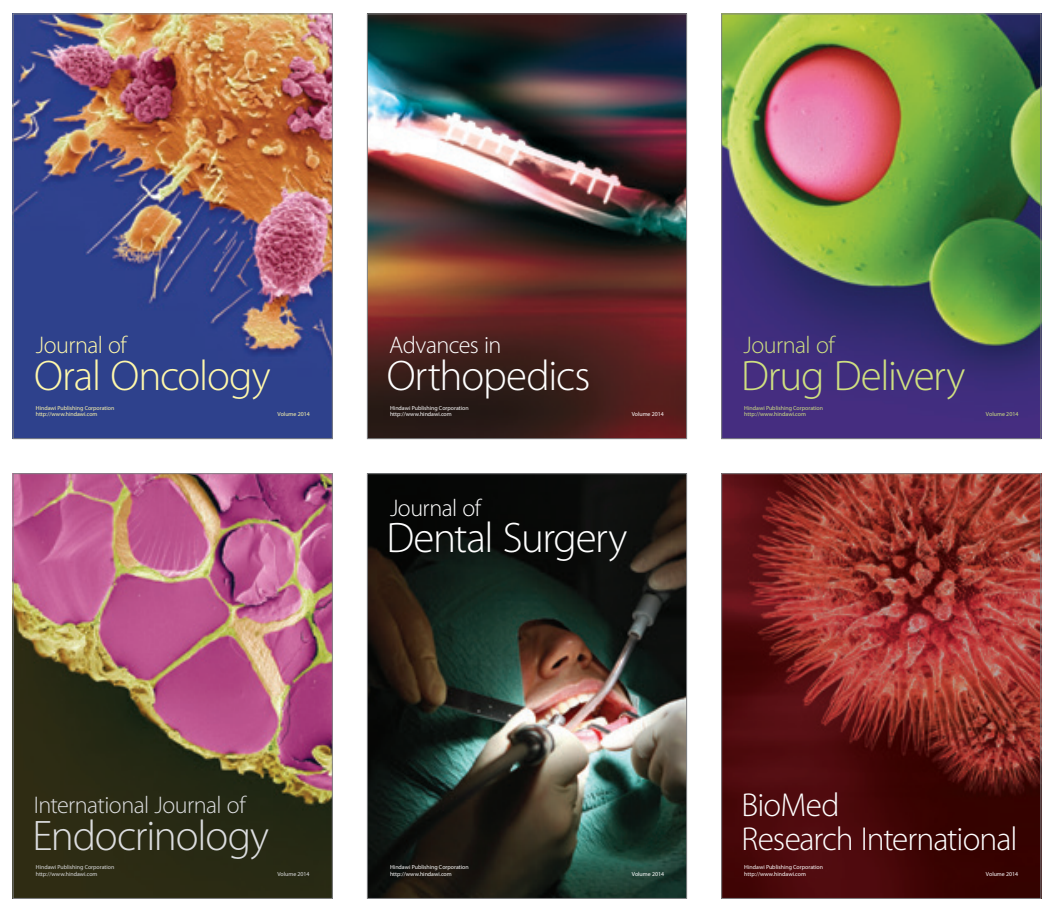

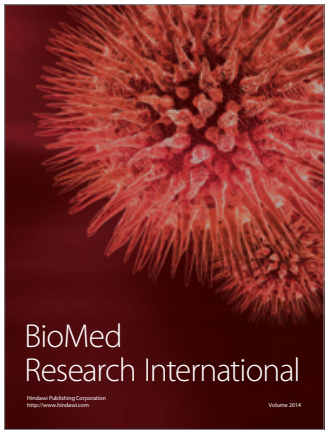

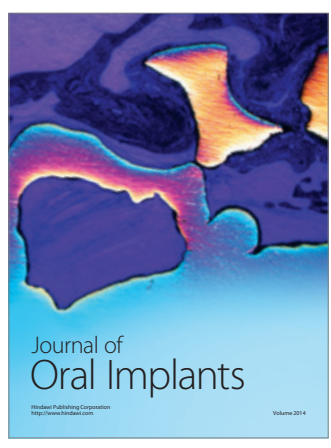
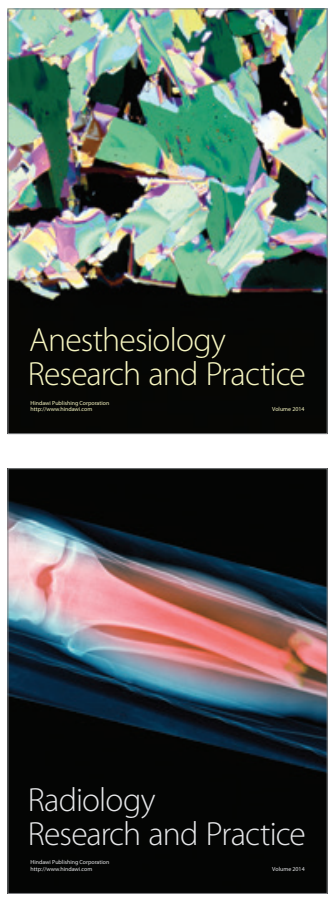